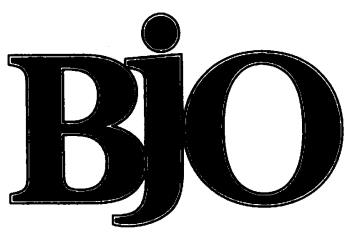

British Journal of Ophthalmology

\title{
Editorials
}

\section{Visual function in myopia without central retinal degeneration}

Is there an additional loss of visual function in myopia beyond that attributable to refractive error and blur of the retinal image? Is there a component of visual loss caused by retinal changes? It is well known that in degenerative myopia, the pathological changes include retinal atrophy and may be associated with abnormalities of the electroretinogram and dark adaptation. ${ }^{1}$ The paper by Mäntyjärvi and Tuppurainen ( $p$ 105) addresses the question of loss of visual function as measured by colour vision and dark adaptation in high myopia without central retinal degeneration. In a group of myopes with refractive errors ranging from -6 to $-29 \mathrm{D}$ and without visible degenerative fundus changes they found a reduction in visual function suggesting minimal impairment of the photoreceptor layer. Although these effects were small and within the normal range, visual function was significantly worse than in the controls.

The underlying anatomical and physiological changes which may contribute to reduced function are not known and may be a combination of factors. Reduced dark adapted sensitivity would be expected to include optical factors as well as retinal components. For example, the use of a lens to correct refractive error at the retina requires that the light incident at the cornea is divergent and this results in less light passing through the pupil. At the retina the size of the retinal image would be reduced and spatial summation may play a role. The magnitudes of these effects can be calculated and reasonably estimated. This would then allow an assessment of the possible contribution of retinal factors. In this study, measurements of axial length were not available so it is not known to what extent stretching of the retina may have contributed. In the chick experimental myopia model, it has been found that expansion of the retinal pigment epithelium occurs which is attributable to passive stretch or growth of existing cells. ${ }^{2}$ Similarly, stretch of the retina could result in a reduction of the density of the photoreceptor mosaic. It would be of interest to know whether there is a reduction of the density of rhodopsin per unit area which could contribute to dark adapted threshold elevations. Investigations with more sensitive tests of visual function may clarify the extent and degree of any loss. For example, abnormalities of dark adaptation may be localised to certain regions of the retina and investigation of function outside the central zone may show greater effects. Similarly, stretch of the retina may result in blue cone sensitivity loss.

The alterations of visual function reported here are very small and would have little significance for most visual tasks. Further study of visual function in myopes may provide an explanation of underlying mechanisms of any losses of sensitivity which are found. This may have some future clinical application in differentiating between purely physiological optical phenomena and precursors to retinal degenerative changes in myopia. However, the main interest of these investigations is in understanding the underlying physiology which leads to loss of visual function in myopia and this may clarify the role of measurements of blue cone function and dark adapted rod function in other conditions.

Department of Visual Science,

F W FITZKE

Institute of Ophthalmology,

University of London,

11-43 Bath Street,

London ECIV 9EL

1 Blach RK, Jay B, Kolb H. Electrical activity of the eye in high myopia. $\mathrm{Br} \mathcal{f}$ Ophthalmol 1966; 50: 629-41.

2 Lin T, Grimes PA, Stone RA. Expansion of the retinal pigment epithelium in experimental myopia. Vis Res 1993; 33: 1881-5. 